\title{
Two-Level Systems with Broken Inversion Symmetry ${ }^{\dagger}$
}

\author{
Giovanni Scala ${ }^{1,2}$ \\ 1 Department of Physics and INFN, University of Bari, I-70126 Bari, Italy; giovanni.scala@ba.infn.it; \\ Tel.: +39-3204681707 \\ 2 Department of Mathematics, University of Bari, I-70126 Bari, Italy \\ + Presented at the 11th Italian Quantum Information Science conference (IQIS2018), Catania, Italy, \\ 17-20 September 2018.
}

Published: 20 November 2019

\begin{abstract}
This research analyzes the impact of the asymmetry of the quantum system and the range of control possibilities offered by dispersive media and the nanostructures. I investigated the asymmetric behavior of a two level system in the Jaynes-Cummings model (JC) and in the spontaneous emission process discussing respectively the presence of a non trivial emission at the Rabi frequency and the correction at a much more basic level, i.e., in the lifetime of the excited state, and also affect the value of the Lamb shift with broken symmetry in the diagonal terms of dipole moment.
\end{abstract}

Keywords: Jaynes-Cummings model; Green tensor propagator; spontaneous emission; permanent dipole moment

\section{Introduction}

With the advent of new technologies, concerning quantum phenomena and nanophotonics, new needs have emerged that have as a goal a greater control over the fundamental devices that form the basis of quantum technologies. This article exploit conditions in which the spatial structure of atomic systems (molecules, defects or quantum dots) is relevant: the fact that these systems are not point-like but they have finite spatial dimensions permits to characterize them by different types of symmetry or, on the contrary, these systems can break the ideal spatial symmetry characteristic of atoms. Therefore finite spatial dimensions combined with a lack of symmetry means that the electric charge distribution in the system's eigenstates becomes important. The charge distribution is characterized in terms of permanent multipole moments, which are responsible for extremely interesting promising effects in terms of their technological potential. Asymmetric quantum systems, characterized by a permanent electrical dipole moment, show in the Jaynes-Cummings model the possibility to be used as optically tunable radiation sources in the range from $\mathrm{GHz}$ to $\mathrm{THz}$ [1]. Moreover, we show how the lifetime of the excited state and the Lamb shift can be modified in the presence of the asymmetry. Under typical conditions, in a regime of weak coupling with a photonic vacuum, this effect is however negligible. In my opinion, dispersive media, and in particular nanostructures built of them, allowing to tailor local density of states of the environment, can enhance these effects by many orders of magnitude and pave the way towards exploiting their potential for new phenomena.

\section{Spontaneous Emission with Asymmetric Dipole}

In the framework of Jaynes-Cummings model which describes the oscillating behavior of a two-level quantum system in a cavity, interacting with one resonant mode of the electromagnetic radiation at an optical frequency a non trivial emission at $\mathrm{THz}$ regime comes up owing to the broken symmetry in the diagonal terms of dipole moment. The model reads as $H_{J C}=H_{a}+H_{f}+H_{i}$, with 


$$
H_{a}=\frac{\hbar \omega_{a}}{2} \sigma_{z}, \quad H_{f}=\hbar \omega_{c} a^{\dagger} a, \quad H_{I}=g_{r}\left(\sigma^{+} \otimes a+\sigma^{-} \otimes a^{\dagger}\right) .
$$

$H_{a}$ is the simplified Hamiltonian for the atomic transitions with energy $\hbar \omega_{a}, \sigma_{z}$ is the third Pauli matrix, $\sigma^{+}=|e\rangle\langle g|$, and $\sigma^{-}=|g\rangle\langle e| . H_{f}$ describes the Hamiltonian of the electromagnetic field with $a$ and $a^{\dagger}$ representing the annihilation and creation operators of one normalized mode in the cavity with frequency $\omega_{c}$, since the commutator of $a$ and $a^{\dagger}$ is the identity. The interaction term $H_{I}$ is written in rotating wave approximation, the simplest coupling of dipole moment and it is quantified by the coupling constant $g_{r}$ which is proportional to the Rabi frequency of the system, as well as, the radiation for diagonalizing the Hamiltonian [2]. In this way the counter-rotating terms are neglected and the dynamics consists of the emission from excited to the ground state by $\sigma^{-}$acquiring a photon by $a^{\dagger}$ and the opposite case where the excitation of the atom from $|g\rangle$ goes to $|e\rangle$ by $\sigma^{+}$and absorbing the photon by the annihilation operator $a$. In order to introduce the asymmetry the dipole operator in the basis $\{|e\rangle,|g\rangle\}$ follows

$$
\boldsymbol{d}=\left(\begin{array}{ll}
\boldsymbol{d}_{e e} & \boldsymbol{d}_{e g} \\
\boldsymbol{d}_{g e} & \boldsymbol{d}_{g g}
\end{array}\right)=\left(\boldsymbol{d}_{e e}+\boldsymbol{d}_{g g}\right) \frac{\boldsymbol{I}}{2}+\left(\boldsymbol{d}_{e e}-\boldsymbol{d}_{g g}\right) \sigma_{z}+\boldsymbol{d}_{e g} \sigma^{+}+\boldsymbol{d}_{g e} \sigma^{-}
$$

Without the diagonal terms only the transitions for $n_{f}=n_{i}-1$ are allowed by rotating wave approximation, but at the first order perturbation theory other transitions at double frequencies of the cavity become opened for the presence of permanent dipole moment [3]. Analytical results for the time-averaged radiation intensity in relevant physical situations are obtained at the first perturbation order in the symmetry violation parameter showing that this effect is more relevant comparing with the counter-rotating terms. Since the Rabi frequency is proportional to the strength of the coupling with the electromagnetic field, the effect can be used for frequency-tuned parametric amplification and generation of electromagnetic waves in the regime of $\mathrm{THz}$ where this transitions are allowed.

Beyond JC, spontaneous emission processes are studied in the presence of asymmetry. Basically the model is the following

$$
H=H_{a}+H_{f}+H_{I}
$$

where for $t=0$ we have

$$
H_{a}=\hbar \omega_{A} \sigma^{\dagger} \sigma, \quad H_{f}=\hbar \int d^{3} r \int d \omega \omega f^{\dagger}(r, \omega) f(r, \omega), \quad H_{I}=-\boldsymbol{d} \cdot \boldsymbol{E}\left(r_{A}\right),
$$

with $\boldsymbol{d}$ comes from Equation (2) and

$$
E_{k}\left(\boldsymbol{r}_{A}, t\right)=i \sqrt{\frac{\hbar}{\pi \epsilon_{0}}} \int_{-\infty}^{\infty} d \omega \frac{\omega^{2}}{c^{2}} \int d^{3} \boldsymbol{r}^{\prime} \sqrt{\Im \epsilon\left(\boldsymbol{r}^{\prime}, \omega\right)} G_{k j}\left(\boldsymbol{r}_{A}, \boldsymbol{r}^{\prime}, \omega\right) f_{j}\left(\boldsymbol{r}^{\prime}, \omega, t\right) .
$$

The index $k$ is the spatial component of the electric field evaluated at the position of the atom and depending on the imaginary part of the permittivity of a non-homogeneous and dispersive medium The evolution is due to the operators $f(\boldsymbol{r}, \omega, t)$ which satisfied the bosonic operator commutation relation

$$
\left[f_{k}(\boldsymbol{r}, \omega), f_{\boldsymbol{k}^{\prime}}\left(\boldsymbol{r}^{\prime}, \omega^{\prime}\right)\right]=\delta_{k k^{\prime}} \delta\left(\boldsymbol{r}-\boldsymbol{r}^{\prime}\right) \delta\left(\omega-\omega^{\prime}\right)
$$

One of the most important outcome is obtained thanks to the resolvent method [4,5], therefore the dressed excited state reads

$$
R_{e}(z)=\sum_{|s, i\rangle} \frac{\left\langle e ; 0\left|H_{\text {int }}\right| s, i\right\rangle\left\langle s, i\left|H_{\text {int }}\right| e ; 0\right\rangle}{z-E_{i}} .
$$


Close the real axis $z=E+i \eta$, therefore $R_{e}(E)=\lim _{\eta \rightarrow 0^{+}} R_{e}(E+i \eta)$ which is

$$
R_{e}(E)=\hbar \Delta_{e}^{(d)}(E)-i \hbar \frac{\Gamma_{e}^{(d)}(E)}{2}+\hbar \Delta_{e}^{(o d)}(E)-i \hbar \frac{\Gamma_{e}^{(o d)}(E)}{2} .
$$

The damping term $\Gamma_{e}^{(d)}(E)$ and the Lamb shift $\Delta_{e}^{(d)}(E)$ are particular interesting because depend on the diagonal terms $\boldsymbol{d}_{e}$ of the dipole matrix, that we have introduce in the our model beyond JC. After a straightforward calculation we get

$$
\begin{gathered}
\Gamma_{e}^{(d)}(E)=d_{e_{i}} d_{e_{j}} \frac{1}{2 \epsilon_{0} \hbar c^{2}} \frac{\left(E_{e}-E\right)^{2}}{\hbar^{2}} \Im G_{i j}\left(\boldsymbol{r}_{A}, \boldsymbol{r}_{A}, \frac{\left(E-E_{e}\right)}{\hbar}\right) \theta\left(E-E_{e}\right), \\
\Delta_{e}^{(d)}(E)=d_{e_{i}} d_{e_{j}} \frac{1}{\pi \epsilon_{0}} P V \int_{0}^{+\infty} d \omega^{\prime} \frac{1}{\left[E-\left(E_{e}+\hbar \omega^{\prime}\right)\right]} \frac{\omega^{\prime 2}}{c^{2}} \Im G_{i j}\left(\boldsymbol{r}_{A}, \boldsymbol{r}_{A}, \omega^{\prime}\right) .
\end{gathered}
$$

The quantity $G$ is the Green tensor propagator which describes the propagation of the external electromagnetic field in a non homogeneous and isotropic medium. It is twice contracted with the permanent dipole vector $\boldsymbol{d}_{e}$ and defined by the following differential equation

$$
\left[\partial_{i} \partial_{m}-\delta_{i m}\left(\partial^{2}+q^{2}(\omega)\right)\right] G_{i j}\left(\boldsymbol{r}, \boldsymbol{r}^{\prime}, \omega\right)=\delta_{m j} \delta\left(\boldsymbol{r}-\boldsymbol{r}^{\prime}\right) .
$$

The most general solution is

$$
G_{i j}\left(\boldsymbol{r}, \boldsymbol{r}^{\prime}, \omega\right)=\left[\partial_{i} \partial_{j}+\delta_{i j} q^{2}(\omega)\right] q^{-2}(\omega) g\left(\left|\boldsymbol{r}-\boldsymbol{r}^{\prime}\right|, \omega\right)
$$

with

$$
g\left(\left|\boldsymbol{r}-\boldsymbol{r}^{\prime}\right|, \omega\right)=\frac{e^{i q(\omega)\left|\boldsymbol{r}-\boldsymbol{r}^{\prime}\right|}}{4 \pi\left|\boldsymbol{r}-\boldsymbol{r}^{\prime}\right|}, \quad q^{2}(\omega)=\frac{\omega^{2}}{c^{2}} \epsilon(\omega) .
$$

In the future we could extend this research for describing the coupling with light or photon vacuum in conditions when it is necessary to go beyond the dipole approximation. This approximation is based on the assumption of a constant value of the electric field in the spatial area occupied by the quantum system. In the empty space or in the macroscopic resonance cavity, the dipole approximation is very well justified due to the significant difference in the wavelength of light and the size of the atomic system. However, the analysis of the photon field modes at least an order of magnitude below the wavelength may require going beyond this approximation. Finally, exploiting photonic nanostructures to control atomic coupling to light, we expect to develop new methods for preparing and controlling atoms in structured environments, e.g., vacuum, electromagnetically isotropic and non-homogeneous media, resonant laser cavities, nanostructures such as waveguides or nanoparticles.

\section{Conclusions}

The research will be generalized on the study of the behavior of quantum systems in interaction with environments with different structure and degree of controllability, including electromagnetic fields in cavities or dispersive media, focusing on the role of the asymmetric charge distribution of the quantum systems, as well as an intrinsic electric dipole moment. The results of this work will have both fundamental interest, since the effect of asymmetry on free and controlled dynamics is analyzed, and relevance for applications, since they will provide new indications on the possibility to control, by properly choosing the environmental properties, the dynamics of systems that are interesting for quantum information and computation. Examples of such surroundings are homogeneous media characterized by dispersion and associated absorption, or nanoscopic objects such as plasmonic or dielectric nanostructures. 
Acknowledgments: This work is supported by Istituto Nazionale di Fisica Nucleare (INFN) through the project "QUANTUM".

Conflicts of Interest: The author declares no conflict of interest.

\section{References}

1. Kibis, O.V.; Portnoi, M.E. Carbon nanotubes: A new type of emitter in the terahertz range. Tech. Phys. Lett. 2005, 31, 671-672.

2. Rempe, G.; Walther, H.; Klein, N. Observation of quantum collapse and revival in a one-atom maser. Phys. Rev. Lett. 1987, 58, 353.

3. Kibis, O.V.; Slepyan, G.Y.; Maksimenko, S.A.; Hoffmann ,A. Matter Coupling to Strong Electromagnetic Fields in Two-Level Quantum Systems with Broken Inversion Symmetry. Phys. Rev. Lett. 2009, 102, 023601.

4. Facchi, P.; Pascazio, S. La Regola d'oro di Fermi; Bibliopolis: Quaderni di Fisica Teorica,Via Arangio Ruiz, Napoli, Italy 1999.

5. Cohen-Tannoudji, C.; Dupont-Roc, J.; Grynberg, G. Atom-Photon Interactions: Basic Processes and Applications; Wiley: Chichester, UK, 1998.

(C) 2019 by the authors. Licensee MDPI, Basel, Switzerland. This article is an open access article distributed under the terms and conditions of the Creative Commons Attribution (CC BY) license (http:/ / creativecommons.org/licenses/by/4.0/). 